\title{
Utility of Limited Protocol Magnetic Resonance Imaging Lumbar Spine for Nerve Root Compression in a Developing Country, Is It Accurate and Cost Effective?
}

\author{
Kiran Hilal, Zafar Sajjad, Raza Sayani, Dawar Khan \\ Department of Radiology, Aga Khan University Hospital, Karachi, Pakistan
}

\begin{abstract}
Study Design: Cross sectional study.
Purpose: To determine the accuracy of the screening magnetic resonance study of the lumbar spine in the diagnosis of nerve root compression in cases of low back pain as compared to the routine magnetic resonance imaging (MRI) study of the lumbar spine.

Overview of Literature: No local study has been conducted for this purpose. In an international study, the reported sensitivity and specificity of screening MRI lumbar spine protocol in the detection of nerve root compression are $54 \%$ and $100 \%$ respectively.

Methods: Patients of both genders older than 20 years of age with low back pain of any duration or any severity who were referred to the radiology department of Aga Khan University Hospital for MRI of their lumbar spine were evaluated. Two sets of MRI imaging were recruited for each patient: one labeled as 'screening' and the other labeled as 'routine'. The findings of screening MRI were compared with the findings of the routine MRI study.

Results: A total of 109 patients fulfilling the inclusion criteria were included in this study. The diagnostic accuracy, specificity and sensitivity of the screening protocol in our study was $100 \%, 100 \%$ and $100 \%$, respectively in comparison with the routine MRI lumbar spine study for the detection of nerve root compression.

Conclusions: Our data proved that the MRI screening study is a highly accurate tool, and its findings are comparable to the routine study for the detection of nerve root compression especially in cases of lumbar spondylosis.
\end{abstract}

Keywords: Lumbar spine magnetic resonance imaging (MRI); Nerve root compression; Screening MRI; Radiculopathy; Disc herniation

\section{Introduction}

Low back pain is one of the most common health problems affecting $2 / 3 \mathrm{rd}$ of the adult population at some stage in their life. This accounts for more sick leaves and disabilities than any other single medical condition. The management of patients with low back pain, either surgi- cal or conservative, depends on the presence and severity of nerve root compression (NRC) [1]. Siddiqui et al. [2] reported the prevalence of NRC in patients with low back pain to be $73 \%$.

Routine magnetic resonance imaging (MRI) of the lumbar spine (which includes sagittal T1-weighted [T1W] and T2W, sagittal T2W with fat saturation, axial

Received May 9, 2012; Revised Dec 19, 2012; Accepted Dec 27, 2012

Corresponding author: Raza Sayani

Radiology Department, Aga Khan University Hospital,

Stadium Road, PO Box 3500, Karachi 74800, Pakistan

Tel: +923458200579 Fax:+922134934294,E-mail: sayani_raza@yahoo.com 
$\mathrm{T} 2$ and myelography sequences) is the modality of choice for the diagnosis of NRC in patients with low back pain. The reported sensitivity and specificity of routine MRI for the detection of NRC are $80.65 \%$ and $100 \%$, respectively [3].

The disadvantages of routine MRI are cost, availability and imaging time compared with other imaging modalities. Therefore, an alternate study which is more time efficient, cost effective, and produces acceptable results is required [4].

A limited MRI of the lumbar spine includes only axial T2 and sagittal T2-weighted images and can be performed in a matter of minutes. This may be referred to as the screening study.

In comparison to routine lumbar spine MRI, the screening study not only reduces the scanning time but also reduces the cost by up to $80 \%$ as compared to routine exams [5]. No data from South East Asia is available to validate its diagnostic accuracy. However, in a study by Chawalparit et al. [3], the sensitivity and specificity of screening MR examination for the detection of NRC were reported to be $54 \%$ and $100 \%$, respectively.

In order to provide cost effective and evidence based patient care, the provision of an efficient imaging modality in patients with low back pain is needed especially in developing countries like Pakistan.

\section{Objective}

To determine the accuracy of the limited protocol of MRI of the lumbar spine in the diagnosis of NRC in cases of low back pain confirmed by routine MRI of the lumbar spine.

\section{Materials and Methods}

This study was conducted at the Department of Radiology, Aga Khan University Hospital from February 2008 to June 2008. The sample size was calculated by taking the estimated prevalence of NRC of 73\% [2] and the reported sensitivity of MRI screening study for nerve compression of $54.84 \%$ [3], and the specificity of $100 \%$. Thus, the bound of error was $10.4 \%$ with a $p$-value $<0.05$; the minimum calculated sample size was 92 patients. This was a cross sectional study with non probability, purposive sampling.

Patients of both genders $>20$ years of age with low back pain of any duration or any severity who were referred to the radiology department of Aga Khan University Hospital for MRI lumbar spine were included in the study.

Those patients with a previous history of lumbar spine surgery or those already diagnosed with NRC and undergoing follow up scans were excluded from the study.

\section{Nerve root compression}

On MRI was considered in the presence of any one or more of the following: 1) Obliteration of the epidural fat plane by the herniated disk material in the medial, lateral, or both aspects of the nerve root which is normally seen as white/bright on T2-weighted axial images. 2) Any change in the thickness of the nerve root compared with the thickness of the contra lateral nerve root. 3) Any displacement of the nerve root.

\section{Data collection procedure}

Informed consent was obtained by the principal investigator from all patients prior to recruiting the patients for MRI scans. 1.5T MR scanner (Magnetom Avanto, Siemens, Erlangen, Germany) (76/18, I-class), was used. Two sets of MR images were acquired for each patient. One labeled as 'screening' consisted of only axial T2 and sagittal T2 sequences and the other labeled as 'routine' included sagittal T1 and T2 sequences, axial T2, T2 sagittal fat saturated sequences and myelography sequences. The sets were anonymised and coded.

Both studies were interpreted by one experienced radiologist with five years of clinical experience and one resident at different periods of time in a blinded fashion to eliminate reporting bias. Observations were documented separately on the data collection Performa regarding nerve root compression. The findings of the limited MRI study were compared with the findings of the routine MRI study for accuracy.

\section{Data analysis procedure}

Statistical analysis was done using statistical package for social sciences SPSS ver. 16.0 for Windows (SPSS Inc., Chicago, IL, USA). Descriptive analysis was conducted i.e., frequencies and percentages for categorical variables like age, gender, duration of pain, severity of pain, level of NRC in the screening group, level of compression in the 
routine group, additional information other than NRC on limited MRI and additional information other than NRC on routine MRI examination.

Severity of pain was assessed on a visual analogue scale (VAS: 0 , no pain; $1-3$, mild pain; $4-7$, moderate pain; $8-10$, severe pain and $>10$, very severe pain). Stratification was done with regard to age, gender, severity and duration of pain to examine the effect of variable on outcome.

\section{Results}

A total of 109 patients fulfilling the inclusion criteria were included in this study. There were $54(49.5 \%)$ males and $55(50.5 \%)$ females in this study (male: female $=1: 1.01$ ).

The mean ( \pm standard deviation) age was $45.6( \pm 15.9)$ years, with a range of 21 to 80 years. Most patients, $n=45$ (41.3\%) were between 21 to 40 years of age. Severity of pain was measured on a VAS. Seventy $(64.2 \%)$ patients had moderate pain, 38 (34.9\%) had severe and $1(0.9 \%)$ had mild pain. The mean duration of pain was $5.1( \pm 10.1)$ months with a minimum-maximum duration of 1 day to 7 years.

Diagnostic accuracy of limited MRI for the diagnosis of NRC was calculated by comparing it with routine MRI. Out of 109 patients studied, the number of true positive cases for limited MRI was 69 with no false negative cases. The number of true negatives was 41 while there were no false positives. The calculated diagnostic accuracy of screening MRI against routine MRI was 100\%.

Diagnostic accuracy of limited MRI for disc herniation was also calculated by comparing it with routine MRI. The calculated diagnostic accuracy of screening MRI was also $100 \%$.

Degenerative disc herniation was the major cause of NRC. Out of 69 positive cases of nerve root compression, $59(85.5 \%)$ were due to lumbar disc herniation. The remaining 10 positive cases of NRC were because of other causes such as infection, metastasis, vertebral collapse etc.

\section{Discussion}

The most common cause for referral for MRI of the lumbar spine in patients with low back pain is suspected nerve root compression. The management of these patients, whether surgical or conservative, depends on the absence or presence of NRC and its severity.
With the advent of MRI, there has been a change in the management trend. A large number of patients with a strong clinical suspicion of NRC and considered as surgical candidates, fail to demonstrate NRC on MRI and are managed conservatively. This shows that abnormal clinical or neurological findings alone are not good predictors of surgical intervention. Therefore, MRI is the most suitable investigation in any patient with consideration for surgery [6].

The most common causes of NRC are degenerative disk disease and herniation [7]. These patients usually initially undergo plain-X-rays to detect gross abnormalities; however, a study done by Nachemson [8] has shown that the yield of plain films is very low with the disadvantage of ionizing radiation.

MRI of the lumbar spine is the modality of choice in evaluating patients with low back pain with clinical suspicion of NRC because of its multi planer imaging and excellent soft tissue contrast.

The disadvantages of MRI are its cost and prolonged scanning time. Although MRI is widely available in Pakistan, its cost limits its access of a large part of the population.

Screening MRI consists of a few selected sequences from the routine study that are likely to be most useful. Screening MRI is used as a screening tool in patients with low back pain to detect any significant abnormalities. The advantages of screening MRI are time efficient, cost effectiveness, and prevention of the patient from unnecessary radiation exposure. Therefore, screening MRI could be considered as the first line of imaging study for patients with suspicious nerve root compression.

A study done by Jarvik et al. [9] found that patients undergoing limited MRI were more satisfied than patients undergoing plain radiographs in terms of reduced hospital referrals, reduced expenditure on other diagnostic tests, earlier return to work and better life-quality following reassurance. The other attraction of the limited protocol is that this can be used to investigate patients with severe low back pain who are claustrophobic and cannot spend much time in the MRI magnet.

The results of our study showed that limited protocols are reliable in detecting NRC with an accuracy of $100 \%$ against the routine MRI study.

Contrary to our results, studies done by Mullan and Kelly [10] and Chawalparit et al. [3] have shown that the limited protocol was less satisfactory in detecting NRC 
Table 1. Causes of nerve root compression not related to degenerative disc disease

\begin{tabular}{lc}
\multicolumn{1}{c}{ Causes } & No. of cases $(\%)$ \\
Multiple myeloma & $1(0.9)$ \\
Infection & $4(3.7)$ \\
Neurofibromatosis & $1(0.9)$ \\
Metastasis & $4(3.7)$ \\
\hline
\end{tabular}

with sensitivities of only $25 \%$ and $82 \%$ and specificities of $100 \%$ and $80 \%$, respectively. We believe that their results were unsatisfactory because of the limited protocol used at their institutes, which only had a single T2 sagittal sequence as opposed to our limited protocol which consisted of T2-weighted sagittal and axial sequences.

Another similar study done by Robertson et al. [5] used a similar protocol to the one used in our study but their results were also not satisfactory in demonstrating nerve root swelling or compression. We assumed that this was due to the difference in the characteristics of sequences in screening and routine protocol of their department. They used a shorter acquisition time, decreased number of slices and small matrix size in the limited screening sequences, and because of this, spatial resolution and image quality were poorer compared to their routine protocol. In our study, there was no difference in the imaging parameters between the limited and routine protocols, so spatial resolution and images quality were the same for both protocols. We believe our results were better because of this reason (Fig. 1). Additional informations besides nerve compression gained from the screening study were spinal canal stenosis, facet joint arthopathy, ligamentun flavum hypertrophy and lateral recess stenosis. These were readily visualized on $\mathrm{T} 2$-weighted axial images of our screening protocol. Other past studies [3-5] including only sagittal T2 sequences in their screening protocols did not find the reliability to demonstrate the above described additional information and emphasized the need for additional sequences.

In $10(14.5 \%)$ patients, NRC was not related to degenerative disc disease and was secondary to other causes such as pathological fracture, metastasis, neurofibromatosis or infection. We discovered that, in these cases, the limited protocol demonstrated NRC but failed to provide sufficient information, and additional sequences or contrast was required to confirm the exact cause (Table 1). We could not analyze the sensitivity, specificity and accu- racy for these diseases separately due to the small number of cases.

Five patients in our study had abnormal diffuse bone marrow signals because of metastasis and 1 because of multiple myeloma. In these patients, the limited protocol was inconclusive because of the absence of additional images. These lesions were readily demonstrated in the routine MRI study with administration of IV contrast material (Fig. 2).

In our study, 3 patients demonstrated focal abnormal

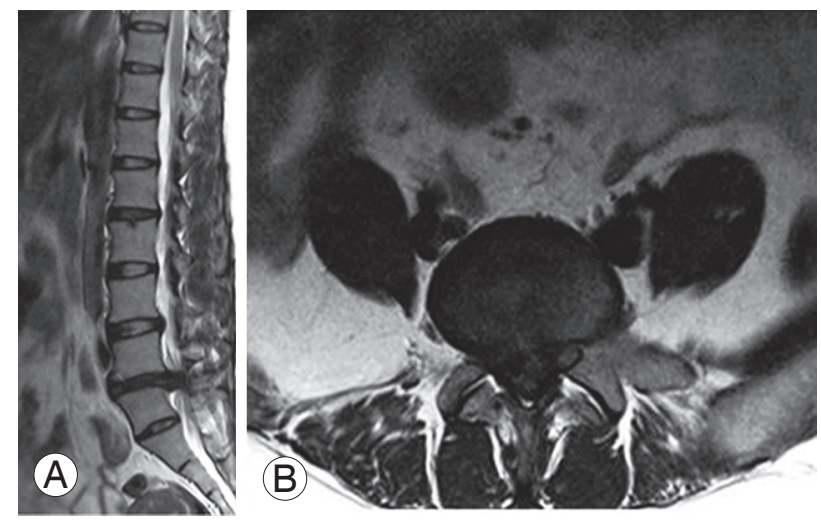

Fig. 1. (A) Sagittal T2. (B) Axial T2-weighted images showing large disc extrusion at the level of $L 4 / L 5$ causing severe bilateral lateral recesses stenosis and narrowing of the canal identified with right neural foraminal stenosis and nerve root compression.

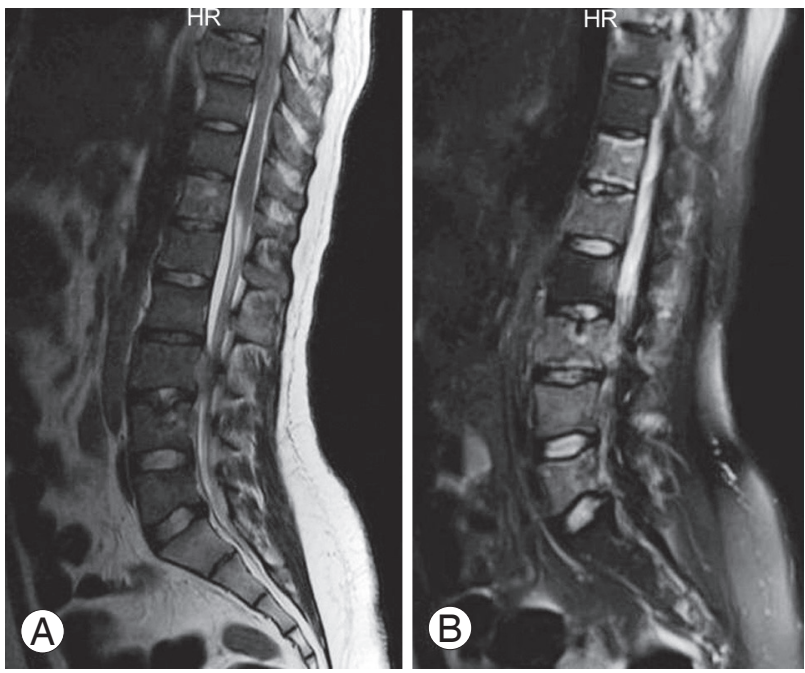

Fig. 2. (A) T2 sagittal image shows abnormal non-conclusive T2 hyperintense signal areas in vertebral bodies at multiple levels with abnormal epidural component at L3 level. (B) Post contrast T1 sagittal fat sat image shows enhancement of corresponding areas with enhancing epidural component causing nerve and cord compression. Findings were secondary to metastatic disease from primary Ewing's sarcoma. HR means head first with right side image. 
signals in the vertebral bodies and were inconclusive. After reviewing theses areas on T1-weighted images of the routine study, theses were confirmed to be hemangiomas (Fig. 3).

The evaluation of hemangioma was not possible without the corresponding T1-weighted sequences. Four patients in our study had abnormal disc signals, surrounding abnormal soft tissue and epidural component. They were inconclusive in the limited protocols. These areas in the routine study after administration of IV contrast material were highly suggestive of infection.

Multiple intra and extra spinal tumors were detected on one patient in the screening study, and the patient was diagnosed with multiple schwannomas in the routine study with IV contrast material.

Patients with suspicious NRC due to malignant, systemic or infectious condition in their clinical history or other clinical tests should be referred for the routine study with contrast rather than undergoing the limited protocol.

Considering nil radiation, cost similar to plain radiography with better sensitivity/specificity in detecting nerve compression compared with plain radiography, screening MRI should be considered as the first imaging modality of choice and should replace plain X-rays in patients with low back pain complicated by radiculopathy. This will help in early diagnosis and also avoid unnecessary expensive and time consuming investigations [9,11].

We also recommend that patients with suspicious or inconclusive signals on the limited protocol should advance the investigation to the routine MRI study.

The routine study should be directly performed in patients with clinical suspicion of malignancy, inflammatory/infectious conditions.

We recognized some limitations of this study. First, we did not have surgical or pathological correlation for our screening protocol findings, instead, we only correlated our findings with the routine study which itself did not have sensitivity greater than $80 \%$. Second, observations were made by a single experienced observer, and because of this, inter observer agreement was not assessed in our study.

Another limitation of our study was that we performed this study on a limited population with low back pain so we could not assess the role of the limited protocol in patients with congenital abnormality e.g., tethered cord or spina bifida who also present the with low back pain and neurological symptoms.

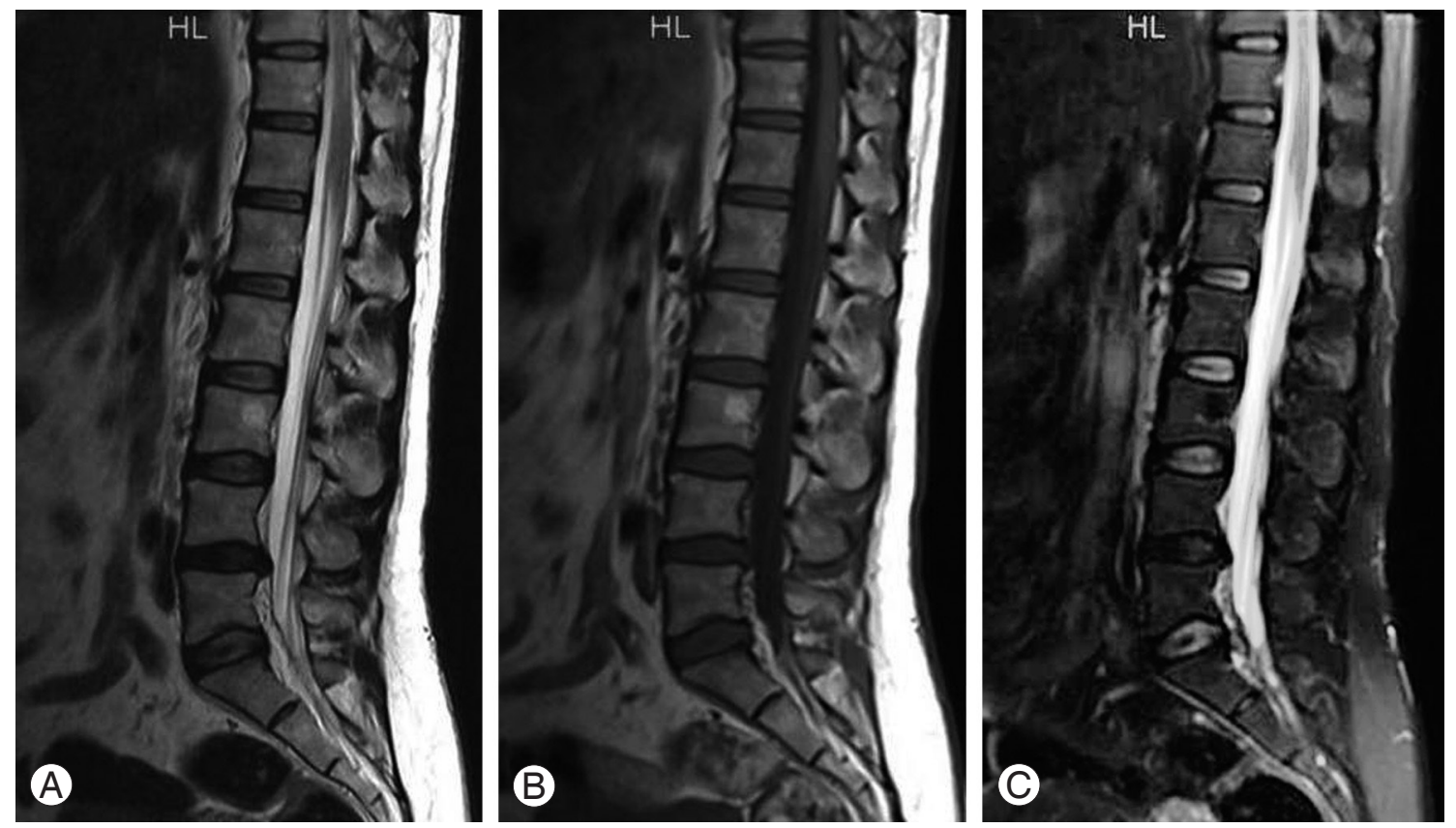

Fig. 3. (A) Sagittal T1 image showing abnormal hyperintense signal at L3 vertebral body. (B) T2-weighted image again redemonstrates hyperintense signal at L3. (C) Fat sat images showing signals drop suggestive of focal fatty deposit. Differentiation was not possible only on screening protocol. HL means Head first with left side image. 


\section{Conclusions}

To summarize, our data confirmed that screening MRI is a highly accurate tool, and its findings are comparable to the routine study for the detection of NRC especially in cases of lumbar spondylosis. Confirmation of other lesions other than disc compression may require routine examination with or without contrast.

\section{Conflict of Interest}

No potential conflict of interest relevant to this article was reported.

\section{References}

1. Jarvik JG, Deyo RA. Diagnostic evaluation of low back pain with emphasis on imaging. Ann Intern Med 2002;137:586-97.

2. Siddiqui AH, Rafique MZ, Ahmad MN, Usman MU. Role of magnetic resonance imaging in lumbar spondylosis. J Coll Physicians Surg Pak 2005;15:396-9.

3. Chawalparit O, Churojana A, Chiewvit P, Thanapipatsir S, Vamvanij V, Charnchaowanish P. The limited protocol MRI in diagnosis of lumbar disc herniation. J Med Assoc Thai 2006;89:182-9.

4. Rankine JJ, Hutchinson CE, Hughes DG. MRI of lumbar spondylosis: a comparison of sagittal T2 weighted and three sequence examinations. Br J Ra- diol 1997;70:1112-21.

5. Robertson WD, Jarvik JG, Tsuruda JS, Koepsell TD, Maravilla KR. The comparison of a rapid screening MR protocol with a conventional MR protocol for lumbar spondylosis. AJR Am J Roentgenol 1996;166:909-16.

6. Hofstee DJ, Gijtenbeek JM, Hoogland PH, et al. Westeinde sciatica trial: randomized controlled study of bed rest and physiotherapy for acute sciatica. J Neurosurg 2002;96:45-9.

7. Tarulli AW, Raynor EM. Lumbosacral radiculopathy. Neurol Clin 2007;25:387-405.

8. Nachemson AL. The lumbar spine: an orthopedic challenge. Spine 1976;1:59-71.

9. Jarvik JG, Hollingworth W, Martin B, et al. Rapid magnetic resonance imaging vs radiographs for patients with low back pain: a randomized controlled trial. JAMA 2003;289:2810-8.

10. Mullan CP, Kelly BE. Magnetic resonance (MR) imaging of lumbar spine: use of a shortened protocol for initial investigation of degenerative disease. Ulster Med J 2005;74:29-32.

11. American College of Radiology. ACR appropriateness criteria. Clinical condition: low back pain [Internet]. Philadelphia: American College of Radiology; 2011 [cited 2013 June 26]. Available from: http://www.acr. org/ /media/ACR/Documents/AppCriteria/Diagnostic/LowBackPain.pdf. 